\title{
Increased expression of HOXB9 in hepatocellular carcinoma predicts poor overall survival but a beneficial response to sorafenib
}

\author{
NAOKAZU CHIBA, YOSUKE OZAWA, KOSUKE HIKITA, MASAAKI OKIHARA, TORU SANO, \\ KOICHI TOMITA, KIMINORI TAKANO and SHIGEYUKI KAWACHI \\ Department of Digestive and Transplantation Surgery, Tokyo Medical University \\ Hachioji Medical Center, Hachioji, Tokyo 193-0998, Japan
}

Received August 31, 2016; Accepted October 31, 2016

DOI: $10.3892 /$ or.2017.5474

\begin{abstract}
At advanced stages of hepatocellular carcinoma (HCC), the multikinase inhibitor sorafenib is the only effective treatment. Surrogate markers that predict the biological and clinical efficacy of sorafenib may help tailor treatment on an individual patient basis. In the present study, the clinical significance of the expression of HOXB9, a transcriptional factor, in HCC was assessed. Increased HOXB9 expression in HCC was found to be positively correlated with the expression of angiogenic factors, increased vascular invasion and was found to be associated with poor overall patient survival. Sorafenib treatment effectively suppressed the expression of angiogenic factors and activation of the Raf/MEK/ERK pathway in HOXB9-expressing HCC cell lines. Consistent with these findings, HCC patients, whose cancer expressed high levels of HOXB9, exhibited increased overall survival upon sorafenib treatment. Collectively, these results suggest that HOXB9 expression in HCC could be a surrogate marker for a beneficial response to sorafenib treatment.
\end{abstract}

\section{Introduction}

HOXB9, a member of the class I homeobox (HOX) genes, regulates several cellular processes, including angiogenesis and maintenance of cell fate (1). It is overexpressed in human breast cancer and its expression is associated with increased

Correspondence to: Dr Naokazu Chiba, Department of Digestive and Transplantation Surgery, Tokyo Medical University Hachioji Medical Center, 1163 Tatemachi, Hachioji, Tokyo 193-0998, Japan E-mail: nchiba0632@yahoo.co.jp

Abbreviations: HOXB9, homeobox B9; HOX, homeobox; VEGF, vascular endothelial growth factor; OS, overall survival; siRNA, short interference RNA; qRT-PCR, quantitative reverse transcription-polymerase chain reaction

Key words: HOXB9, sorafenib, hepatocellular carcinoma, angiogenesis, liver cancer tumorigenicity, lung metastasis and radioresistance $(2,3)$. HOXB9 transactivates several angiogenic factors as well as erythroblastic leukemia viral oncogene homolog (ErbB) and TGF- $\beta$ ligands leading to epithelial-mesenchymal transition (EMT), increased angiogenesis and distal metastasis. In particular, HOXB9 induces tumor proliferation and metastasis by activating angiogenesis; however, little is known concerning the relationship between $\mathrm{HOXB} 9$ expression and angiogenesis in hepatocellular carcinoma (HCC).

HCC is considered a serious public health concern particularly in endemic areas of hepatitis B or C viral infection, including Africa and Southeast Asia (4), despite recent progress in surgical and non-surgical treatment including multikinase inhibitor therapy.

The prognosis for HCC patients is still dismal, since the disease is discovered at an advanced stage in a substantial number of patients when curative therapy is no longer possible (5). Curative treatments (e.g., resection, transplantation or local ablation) are available only to patients with earlystage disease, however they are limited by high recurrence rates, impairing patient outcomes. At advanced stages, the multikinase inhibitor sorafenib is the only effective treatment, although vigorous efforts are underway to better characterize the molecular pathogenesis of liver cancer to refine the efficacy of other molecular-targeted therapies (6).

Sorafenib (Nexavar ${ }^{\circledR}$; Bayer HealthCare Pharmaceuticals, Montville, NJ, USA) is an oral multikinase inhibitor that inhibits the serine-threonine kinases Raf-1 and B-Raf, the receptor tyrosine kinase activity of vascular endothelial growth factor (VEGF) receptors 1-3, and platelet-derived growth factor receptor $\beta$ (7). It blocks tumor cell proliferation and tumor angiogenesis, and increases the rate of apoptosis in a wide range of tumor models by targeting the Raf/mitogen-activated protein kinase/extracellular signal-regulated kinase (RAF/MEK/ERK) and VEGF signaling pathways. Therefore, identification of accurate prognostic biomarkers to distinguish patients at high risk of recurrence or metastasis and surrogate markers for nonsurgical therapy is of the utmost importance for developing preventive strategies to improve the outcome of HCC patients.

In the present study, we demonstrated that increased expression of HOXB9 in $\mathrm{HCC}$ is associated with the induction 
of angiogenic factors, increased vascular invasion and poor overall survival of these patients. Sorafenib treatment was found to suppress the expression of angiogenic factors and the $\mathrm{Raf} / \mathrm{MEF} / \mathrm{ERK}$ pathway in HOXB9-expressing cells in vitro, and HCC patients with increased HOXB9 expression exhibited increased overall survival upon treatment with sorafenib. Collectively, these results suggest that HOXB9 expression in $\mathrm{HCC}$ could be a surrogate marker for a beneficial response to sorafenib treatment.

\section{Materials and methods}

Cell lines and cell culture. The human HCC cell lines, HepG2 and Hep3B, were obtained from the American Type Culture Collection (ATCC; Manassas, VA, USA). The identity of all cells was independently authenticated by short tandem repeat genotyping in May 2014. The cells were cultured in Dulbecco's modified Eagle's medium (DMEM), supplemented with $10 \%$ bovine fetal serum, $100 \mathrm{U} / \mathrm{ml}$ penicillin, $100 \mu \mathrm{g} / \mathrm{ml}$ streptomycin, $1 \mathrm{X}$ non-essential amino acids and $1 \mathrm{mM}$ sodium pyruvate, and incubated in humidified $37^{\circ} \mathrm{C}$ incubators with $5 \% \mathrm{CO}_{2}$. Chemically synthesized Stealth RNAi ${ }^{\mathrm{TM}}$ siRNAs were purchased from Invitrogen (Carlsbad, CA, USA) and were used to knock down endogenous HOXB9 expression in HCC cells. Two different siRNAs were individually transfected into the cells at a final concentration of $100 \mathrm{nM}$ using Lipofectamine RNAiMAX (Invitrogen). In addition, siGFP was used as a control siRNA.

Ethical approval, consent to participate and consent of publication. The present study was approved by the Ethics Committee of Tokyo Medical University. The patients provided consent for their participation and the publication of the present study.

mRNA expression. Our analysis included $79 \mathrm{HCC}$ patients who underwent consecutive resection between September 2008 and December 2014 at Tokyo Medical University Hachioji Medical Center. All protocols were performed in accordance with the Ethics Committee of Tokyo Medical University. Consecutive frozen tissue specimens containing cancerous and matched normal hepatocyte regions were available from $\mathrm{HCC}$ patients (who received a pathological diagnosis) to assess gene panels by quantitative reverse-transcription PCR (qRT-PCR). HOXB9 mRNA expression was examined in cancer and in normal hepatocytes derived from consecutive samples of patients who had undergone surgery at our institute during 2008-2014. The expression ratios (cancer to normal hepatocytes surrounded by cancer) were examined using qRT-PCR. RNA was extracted from cells using the RNeasy kit (Qiagen, Valencia, CA, USA). Conditions for semi-quantitative amplification of cDNA were $95^{\circ} \mathrm{C}$ for $2 \mathrm{~min}$, followed by 25 cycles of $95^{\circ} \mathrm{C}$ for $30 \mathrm{sec}, 56^{\circ} \mathrm{C}$ for $30 \mathrm{sec}$, and $72^{\circ} \mathrm{C}$ for $60 \mathrm{sec}$, with a final extension cycle at $72^{\circ} \mathrm{C}$ for $10 \mathrm{~min}$. RT-PCR analysis was run in triplicate for each sample on a Light Cycler 480 Real-Time PCR System using SYBR-Green I Master Mix (Roche, Mannheim, Germany). The following program was run: pre-incubation for $5 \mathrm{~min}$ at $95^{\circ} \mathrm{C}$, amplification for 45 cycles $(10 \mathrm{sec}$ of denaturation at $95^{\circ} \mathrm{C}, 10 \mathrm{sec}$ of annealing at $57^{\circ} \mathrm{C}$ and a $10-\mathrm{sec}$ extension at $72^{\circ} \mathrm{C}$ ), with melting curve analysis. mRNA levels of the target genes were normalized against mRNA levels of GAPDH, which was used as an internal control. The sequences of all the primers used are listed as follows: HoxB9 forward, 5'CACCATGTCCATTTCTGGGACGCTTAG3' and reverse, 5'AAACTCTTTGCCCTGCTCCTTATTC3'; GAPDH forward, 5'ATCATCCCTGCCTCTACTGG3' and reverse, 5'TTTCTAGACCGGCAGGTCAGGT3'; PDGF-b forward, 5'GATCCGCTCCTTTGATGATC3' and reverse, 5'GTCTCACACTTGCATGCCAG3'; PDGFR-b forward, 5'AATGTCTCCAGCACCTTCGT3' and reverse, 5'AGCGGA TGTGGTAAGGCATA3'; VEGF-A forward, 5'ACCATGCCA AGTGGTCCCAG3' and reverse, 5'CTTTCTTTGGTCTGCA TTCACA3'; VEGF-C forward, 5'CTTCTTTAAACCTCCATG TGTGTC3' and reverse, 5'GAATGAACTTGTCTGTAAACA TCCA3'; VEGFR-2 forward, 5'TCATCTGTTACAGCTTCC AAGT3' and reverse, 5'GGTTTGATTCTTTCCAGGCTC3'; VEGFR-3 forward, 5'CCAGCATCGTGTGGTACAAAGA3' and reverse, 5'CTCCCCGGGGTCCATGATGAT3'.

Western blotting and immunohistochemistry. Protein expression was detected by western blotting using antibodies against HOXB9 and pERK (phospho-ERK) (Santa Cruz Biotechnology, Santa Cruz, CA, USA); ERK2, pMEK (phospho-MEK) and MEK1 (Cell Signaling Technology, Beverly, MA, USA); and pRAF1, Raf1 and actin (Abcam, Cambridge, MA, USA). The western blotting protocols used have been previously described (8). Immunohistochemistry (IHC) was carried out on $5-\mu \mathrm{m}$ sections of formalin-fixed paraffin-embedded (FFPE) tissue by heat-induced epitope retrieval in $10 \mathrm{mmol} / \mathrm{l}$ sodium citrate $(\mathrm{pH}$ 6.0) before blocking with 5\% BSA-PBS. For IHC, HOXB9 expression was detected using the anti-human HOXB9-specific antibody at a dilution of 1:250 (Santa Cruz Biotechnology).

Sorafenib treatment. Cells were plated into 12- to 24-well tissue culture plates or $10-\mathrm{cm}^{2}$ dishes and cultured overnight. Sorafenib (LC Laboratories, Woburn, MA, USA) was added to the cells at two different concentrations (5 and $1 \mu \mathrm{M})$ for $48 \mathrm{~h}$ before mRNA and protein extraction. The final dimethyl sulfoxide (DMSO) concentration in all experiments was $<0.05 \%$. A total of 15 patients with tumor-node-metastasis (TNM) stage III HCC were treated with sorafenib after curative resection.

Statistical analyses. Data represent the mean \pm standard error of the mean. Comparisons between groups were made using a two-tailed t-test or U test for continuous variables, and Fisher's exact test for comparison of proportions. Correlations were assessed with the non-parametric Spearman's coefficient. All calculations were carried out with the SPSS software package (SPSS 18.0). Overall survival (OS) curves were drawn according to Kaplan-Meier estimates and compared by log-rank tests. $\mathrm{P}<0.05$ was defined as significant.

\section{Results}

HOXB9 expression in HCC. To investigate HOXB9 expression in HCC, we examined samples from $79 \mathrm{HCC}$ patients who received curative resection at our institute. HOXB9 mRNA was expressed in $\sim 40 \%$ of the patients (Fig. 1A) and was 
A
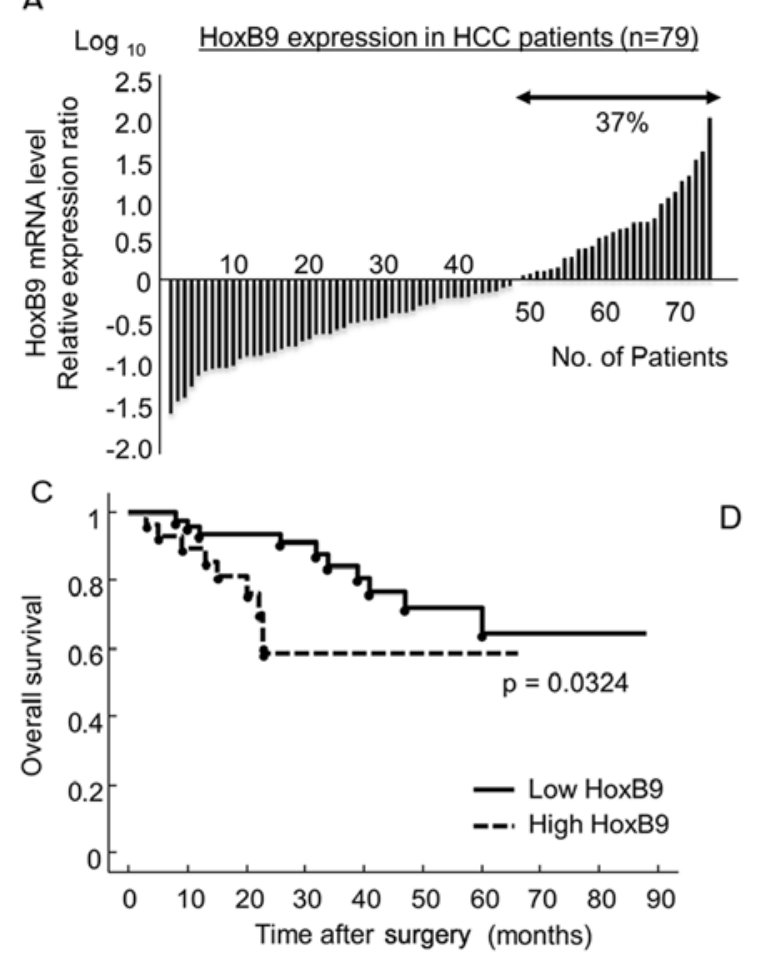

D
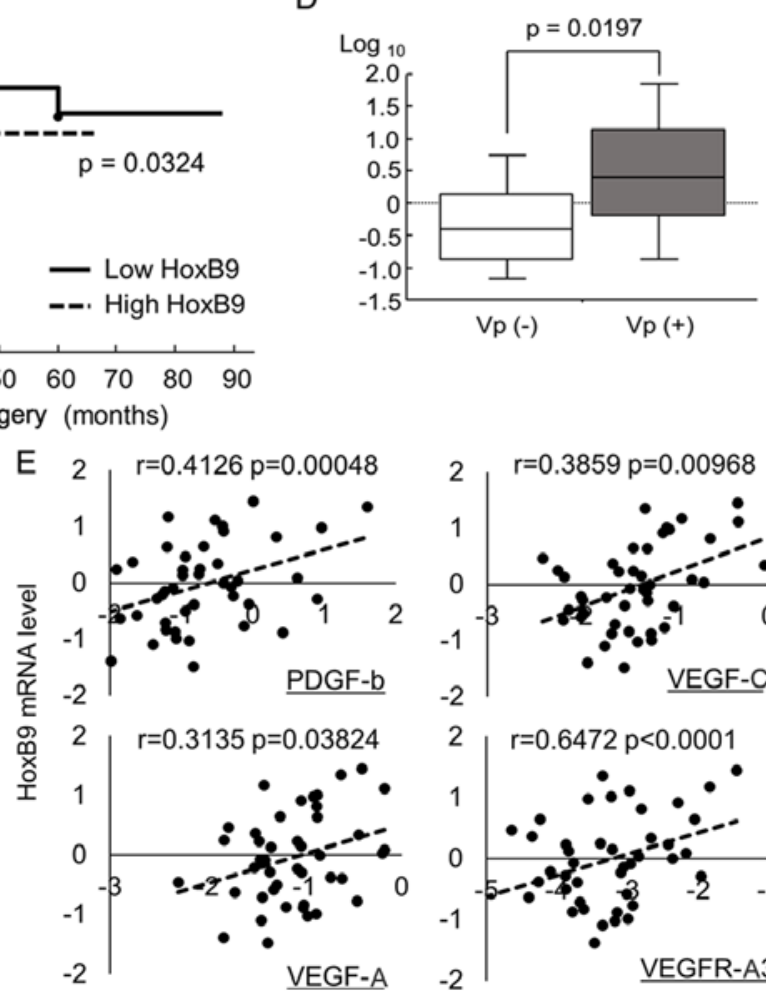

B

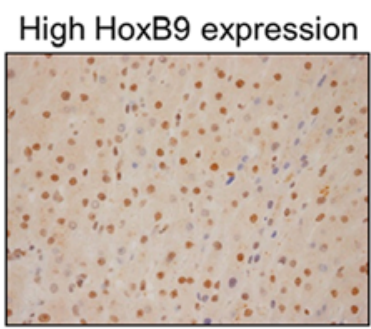

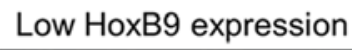

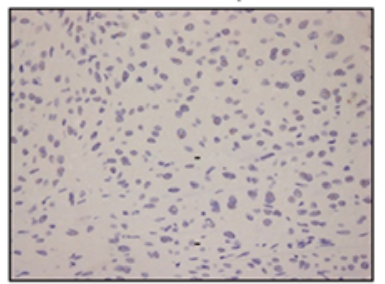

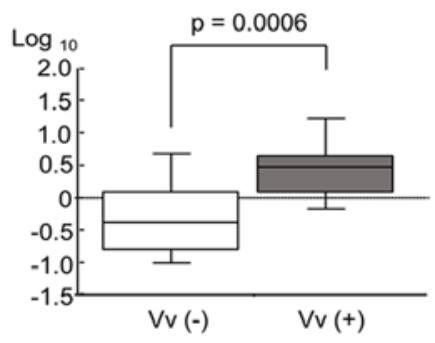

Figure 1. Homeobox B9 (HOXB9) expression in hepatocellular carcinoma (HCC) patients. (A) Waterfall plot of relative mRNA expression levels of HOXB9 in HCC cells compared with these levels in normal hepatocytes, as determined using qRT-PCR in triplicate (n=79 patients). (B) Representative immunohistochemical staining of HOXB9 in resected HCC specimens showing high and low HOXB9 expression. (C) Kaplan-Meier overall survival curve of HCC patients stratified according to HOXB9 mRNA expression ( $n=79$ patients). (D) Relative mRNA expression of HOXB9 in patients according to vascular invasion [left panel, portal vein invasion (Vp); right panel, hepatic vein invasion (Vv)]. Error bars indicate SDs. (E) Correlation plots of HOXB9 mRNA and angiogenic factors PDGF-b, VEGF-C, VEGF-A and VEGFR-A3 as determined using qRT-PCR in triplicate.. P-values were determined using the U test and the log-rank test. $\mathrm{R}^{2}$ values were calculated by correlation test.

upregulated in tumors relative to uninvolved adjacent normal hepatocytes as determined by both qRT-PCR and immunohistochemical staining (Fig. 1B). Although, HOXB9 expression in HCC did not affect disease-free survival after surgery (data not shown), increased HOXB9 expression in HCC was significantly associated with a poor prognosis in OS (5-year OS rate; 63.5 vs. $58.5 \%$; $\mathrm{p}=0.0324)$ (Fig. 1C), as previously reported for breast and colorectal cancer $(9,10)$. Moreover, HOXB9 mRNA expression was significantly higher in patients exhibiting vascular invasion (both portal and hepatic vein) compared to those who were negative for vascular invasion (Fig. 1D). A significant positive correlation was observed between HOXB9 mRNA expression and several angiogenic factors targeted by sorafenib: PDGF-b, VEGF-C, VEGF-C and VEGFR-A3 (p<0.05) (Fig. 1E) (11).
HOXB9 regulates the expression of angiogenic factors and Raf/MEK/ERK signaling. HCC cell lines, HepG2 and Hep3B, showed high HOXB9 mRNA expression relative to the human mammary epithelial cell line, MCF10A, which showed low HOXB9 mRNA (Fig. 2A) as previously reported (2). To determine the effect of the loss of HOXB9 in HCC cells, we depleted HOXB9 mRNA in these cells using two short interfering RNA (siRNA) oligonucleotides (Invitrogen) targeting HOXB9. Both siRNAs decreased HOXB9 expression in the HCC cells by -130 and $-230 \%$ (Fig. 2B). A decrease in HOXB9 expression by these siRNAs also decreased the expression of several angiogenic factors. These angiogenic factors which are targeted by sorafenib were highly expressed in the siGFP-transfected control HepG2 and Hep3B 
A

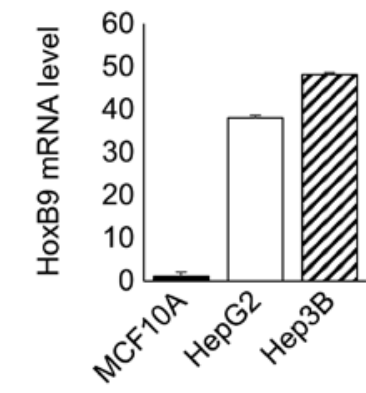

B

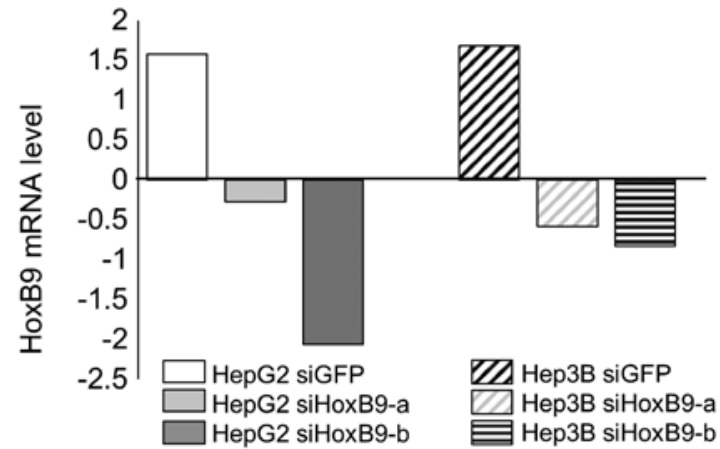

C

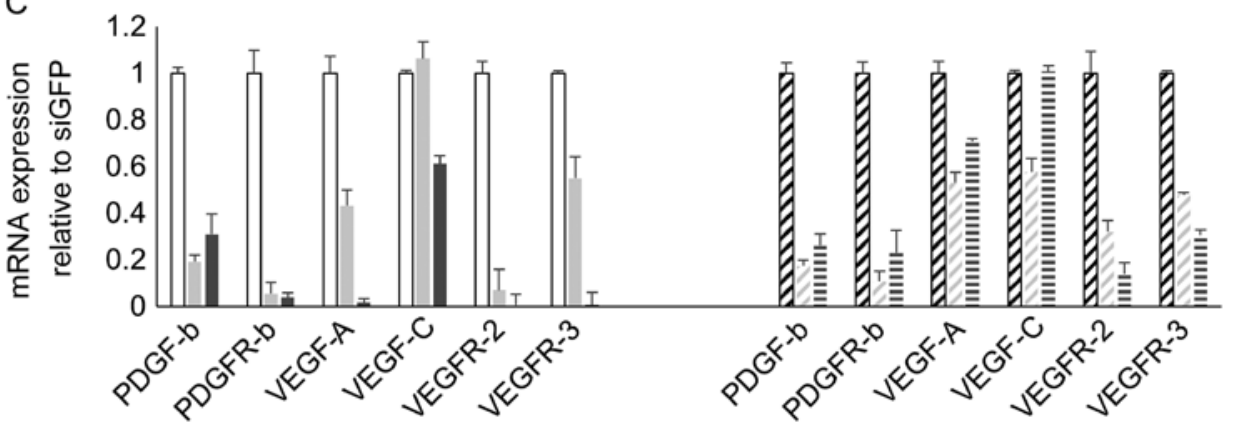

D
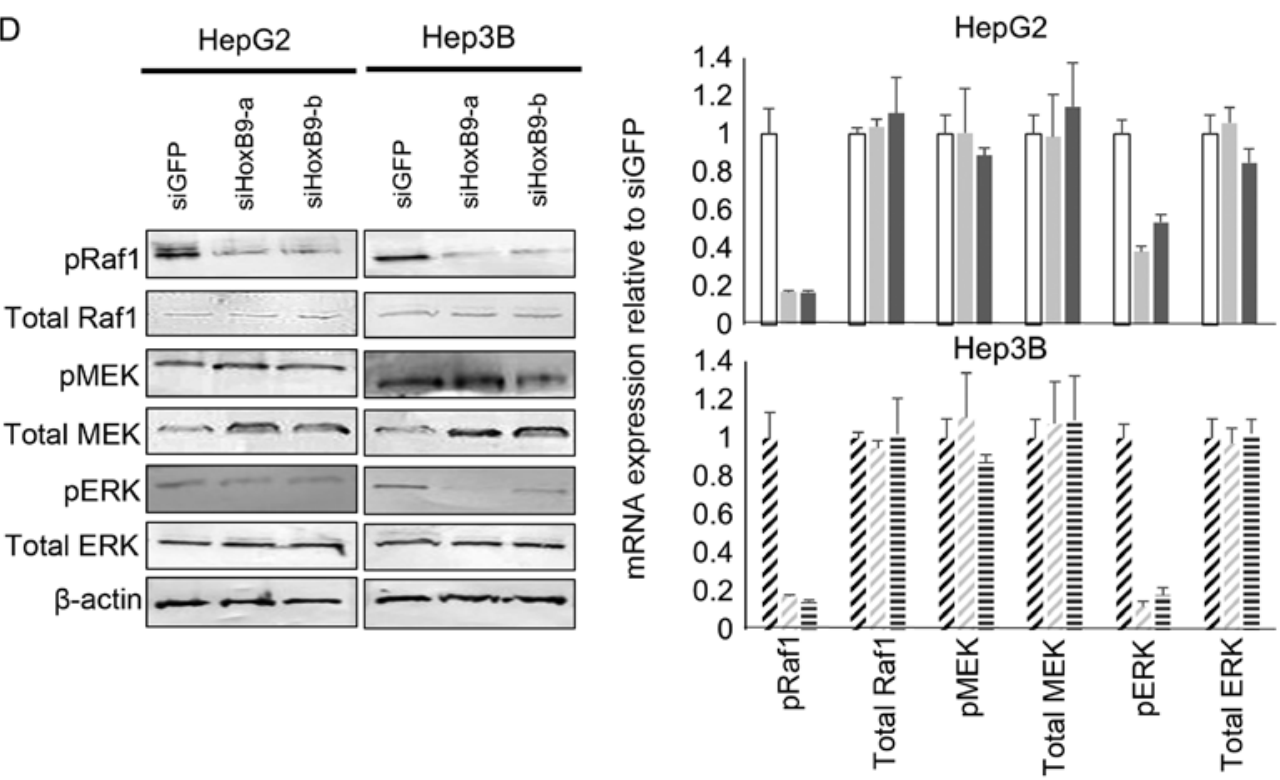

Figure 2. Homeobox B9 (HOXB9) regulates the expression of angiogenic factors and the RAF/MEK/ERK pathway. (A) HOXB9 mRNA expression in hepatocellular carcinoma (HCC) cell lines as determined by qRT-PCR. Expression in MCF10A cells (immortalized normal mammary epithelial line) was used as the control. (B) Knockdown of HOXB9 expression in HCC cell lines following transfection of siRNA against HOXB9. RNA levels were quantified by qRT-PCR (C) Expression of angiogenic factors in siGFP- and siHOXB9-transfected HCC lines. qRT-PCR analysis showed that depletion of HOXB9 suppressed the expression of these angiogenic factors. (D) The Raf/MEK/ERK axis protein expression in HOXB9-knockdown cells, as determined by western blotting. The left panel shows the results of the western blot analysis and the right panel displays the quantification of these results. Error bars indicate SDs. P-values were determined using the log-rank test.

lines (Fig. 2C). These results are consistent with previously reported results in breast cancer $(2,10)$. Moreover, a decrease in HOXB9 expression in the two HCC cell lines also mitigated the Raf/MEK/ERK pathway (Fig. 2D), which is targeted by sorafenib1 (2). Knockdown of HOXB9 expression led to a decrease in phospho(p)Raf and pERK. The quantification of these western blotting bands are shown (Fig. 2D, right panel).

Significance of HOXB9 expression on sorafenib response. To determine whether sorafenib, the multikinase inhibitor suppresses the Raf/MEK/ERK pathway upregulated in HOXB9-expressing cells, we treated the cells transfected with siGFP and siHOXB9 (expressing high or low HOXB9, respectively) with 1 and $5 \mu \mathrm{M}$ of sorafenib. The proteins of the Raf/MEK/ERK pathway were more markedly suppressed in the cells expressing high levels of HOXB9 compared to those expressing low HOXB9 mRNA (Fig. 3A). The quantification of these western blotting bands is shown (Fig. 3B).

We similarly determined whether sorafenib treatment suppresses the expression of HOXB9-regulating angiogenic 


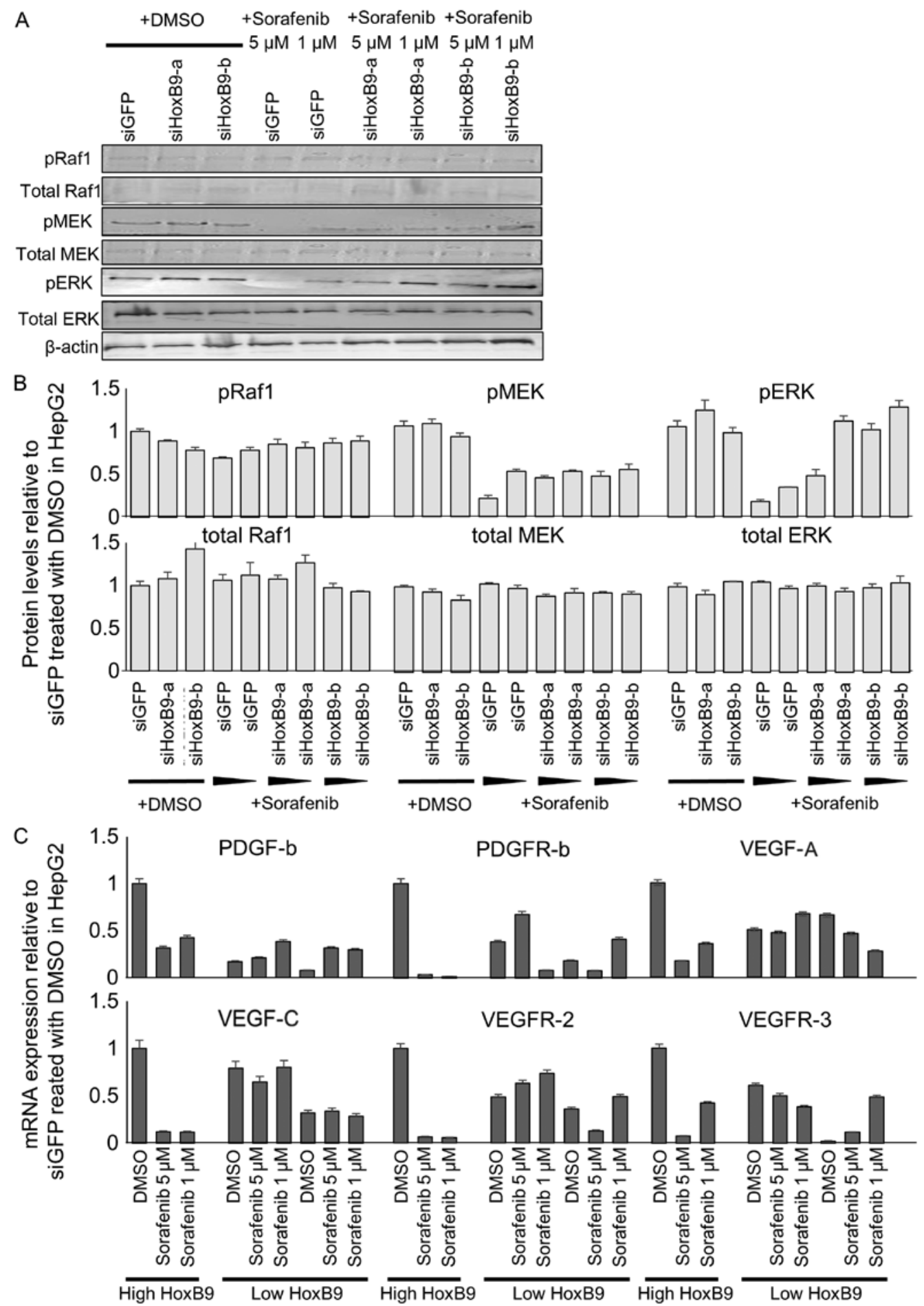

Figure 3. Sorafenib treatment effectively suppresses angiogenic factors in HOXB9-expressing cells in vitro. (A and B) Western blot analysis showed the downregulation of proteins involved in the Raf/MEK/ERK pathway by increasing the concentration of sorafenib. High and low HOXB9-expressing cells were derived by transfecting siGFP and siHOXB9 into cells. DMSO was used as a vehicle control. The upper panel A shows the results obtained from the western blot analysis and the lower panel B shows the quantification of these results. (C) Suppression of mRNAs of angiogenic factors by increasing the concentration of sorafenib as assessed by qRT-PCR in cells expressing high and low levels of HOXB9 (derived from siGFP and siHOXB9 transfection of cells, respectively). DMSO was used as a vehicle control. Data represent 3 independent experiments. Error bars indicate SDs.

factors in cells expressing high and low HOXB9. Sorafenib potently suppressed the expression of several angiogenic factors by $60-95 \%$ in cells expressing high HOXB9 cells compared to the levels in cells expressing low levels of HOXB9 (-50 and $-10 \%)$ (Fig. 3C). Collectively these results show that HOXB9-expressing cells, by expressing higher baseline levels of angiogenic factors and activation of the Raf/MEK/ERK pathway, are more likely to be responsive to sorafenib treatment.

To clarify the clinical significance of these findings, we conducted a retrospective study in HCC patients who underwent resection before treatment with sorafenib at our institute 
A

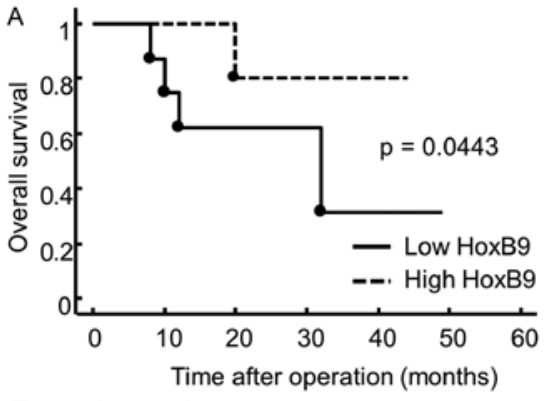

Truncation number

$\begin{array}{llllllll}\text { High } & 6 & 6 & 5 & 5 & 5 & - & - \\ \text { Low } & 9 & 7 & 6 & 6 & 3 & 3 & -\end{array}$

B

\begin{tabular}{lll}
\hline & \multicolumn{1}{c}{$\begin{array}{c}\text { High HoxB9 } \\
(n=6)\end{array}$} & \multicolumn{1}{c}{$\begin{array}{c}\text { Low HoxB9 } \\
(n=9)\end{array}$} \\
\hline Age & 64 & 70 \\
Gender M/F & $5 / 1$ & $9 / 0$ \\
Child A/B & $5 / 1$ & $7 / 2$ \\
Patho well,mod/poorly & $4 / 2$ & $6 / 3$ \\
AFP & $2426(3-144591)$ & $2854(18-17045)$ \\
PIVKA-II & $60898(24-318000)$ & $29304(14-170000)$ \\
\hline
\end{tabular}

Figure 4. Hepatocellular carcinoma (HCC) patients with high homeobox B9 (HOXB9) expression exhibited increased overall survival upon sorafenib treatment. (A and B) HCC patients with high HOXB9 expression treated with sorafenib exhibited increased overall survival. HOXB9 mRNA expression levels were evaluated in triplicate by qRT-PCR in patients who were treated with sorafenib. Kaplan-Meier overall survival curve of HCC patients treated with sorafenib, according to HOXB9 mRNA expression. P-values were determined using the log-rank test.

between 2008 and $2012(n=15)$. Samples obtained from patients before chemotherapy were analyzed by qRT-PCR. The demographic and clinical data showed no significant differences with regard to age, gender, pathological stage or chemotherapy between groups with high and low expression of HOXB9. High expression of HOXB9 mRNA was associated with longer OS after treatment with sorafenib (3-years OS rate; 80 vs. $30 \%$, $\mathrm{p}=0.0443$ ) compared with patients expressing low levels of HOXB9 (Fig. 4A). In addition, clinical findings among the two groups had no significant difference (Fig. 4B).

\section{Discussion}

HOXB9 has been reported to be overexpressed in neoplastic tissues (13). HOXB9 promotes the expression of angiogenic factors, as well as ErbB and TGF- $\beta$ ligands. From a biological perspective, multiple HOX-binding sites are present in the promoters of angiopoietin-like 2 , IL-8, TGF- $\beta 2$, VEGF and bFGF. AREG, ERG, VEGF and bFGF are direct targets of HOXB9-induced transcriptional activation $(14,15)$. In the present study, we demonstrated that HOXB9 promoted the expression of angiogenic factors and the Raf/MEK/ERK pathway in HCC. Both angiogenesis and signaling through the $\mathrm{RAF} / \mathrm{MEK} / \mathrm{ERK}$ cascade play critical roles in the development of HCC and may lead to increased tumorigenesis and poor overall survival in HCC patients.

Sorafenib is a multikinase inhibitor with activity against the Ser/Thr kinase Raf, known to be important in tumor cell signaling and tumor cell proliferation, and in the regulation of several receptor tyrosine kinases involved in angiogenesis, including VEGFR-2 and PDGFR-12. Although the HOXB9 gene has not been described as a direct target of sorafenib, its ability to induce angiogenesis may render HOXB9-expressing HCC cells sensitive to sorafenib. This is consistent with our data showing increased overall survival of HOXB9overexpressing HCC patients treated with sorafenib. There is increasing evidence of the potential crosstalk between, for example, HOXB9 and wingless/T-cell factor (Wnt/TCF) signaling pathways (16). Expression profiling of HCC patients treated with sorafenib may shed light on potential correlations between HOXB9-related molecular classes and treatment responses.

Surrogate biomarkers that predict the biological and clinical efficacy of sorafenib may help tailor treatment on an individual patient basis. The predictive value of the Raf/MEK/ERK signaling activity for the efficacy of sorafenib in HCC remains uncertain $(17,18)$. In the present study, we found a strong correlation between increased HOXB9 activity in $\mathrm{HCC}$ and poor prognosis, but better therapeutic responses to sorafenib. Evaluation of HOXB9 activity in HCC can be useful to differentiate between responders and non-responders before commencing sorafenib treatment and to help select patients who are likely to benefit from the treatment. However, the present study, was based on a retrospective analysis of a small number of patients at a single site, and selection of patients for sorafenib treatment was subjective. The clinical significance of HOXB9 must therefore be addressed in a prospectively planned multicenter trial. Multigene assays involving a large number of specimens before and after sorafenib exposure may provide more reliable insights into tumor biology and the response to sorafenib treatment.

\section{Acknowledgements}

We thank Dr Shyamala Meheswaran (Cancer Center, Massachusetts General Hospital and Harvard Medical School) for the critical reading of the manuscript. This research was funded by a JSPS KAKENHI Grant-in-Aid for Young Scientists (B) (no. 25861217) and by the Tokyo Medical University Cancer Research Foundation.

\section{References}

1. Cantile M, Schiavo G, Terracciano L and Cillo C: Homeobox genes in normal and abnormal vasculogenesis. Nutr Metab Cardiovasc Dis 18: 651-658, 2008.

2. Hayashida T, Takahashi F, Chiba N, Brachtel E, Takahashi M, Godin-Heymann N, Gross KW, Vivanco M, Wijendran V, Shioda T, et al: $H O X B 9$, a gene overexpressed in breast cancer, promotes tumorigenicity and lung metastasis. Proc Natl Acad Sci USA 107: 1100-1105, 2010.

3. Chiba N, Comaills V, Shiotani B, Takahashi F, Shimada T, Tajima K, Winokur D, Hayashida T, Willers H, Brachtel E, et al: Homeobox B9 induces epithelial-to-mesenchymal transitionassociated radioresistance by accelerating DNA damage responses. Proc Natl Acad Sci USA 109: 2760-2765, 2012.

4. Sherman M: Hepatocellular carcinoma: Epidemiology, surveillance, and diagnosis. Semin Liver Dis 30: 3-16, 2010.

5. Rampone B, Schiavone B, Martino A, Viviano C and Confuorto G: Current management strategy of hepatocellular carcinoma. World J Gastroenterol 15: 3210-3216, 2009.

6. Llovet JM, Ricci S, Mazzaferro V, Hilgard P, Gane E, Blanc JF, de Oliveira AC, Santoro A, Raoul JL, Forner A, et al; SHARP Investigators Study Group: Sorafenib in advanced hepatocellular carcinoma. N Engl J Med 359: 378-390, 2008. 
7. Wilhelm SM, Carter C, Tang L, Wilkie D, McNabola A, Rong H, Chen C, Zhang X, Vincent P, McHugh M, et al: BAY 43-9006 exhibits broad spectrum oral antitumor activity and targets the RAF/MEK/ERK pathway and receptor tyrosine kinases involved in tumor progression and angiogenesis. Cancer Res 64: 7099-7109, 2004.

8. Ha TU, Segev DL, Barbie D, Masiakos PT, Tran TT, Dombkowski D, Glander M, Clarke TR, Lorenzo HK, Donahoe PK, et al: Mullerian inhibiting substance inhibits ovarian cell growth through an $\mathrm{Rb}$-independent mechanism. J Biol Chem 275: 37101-37109, 2000.

9. Seki H, Hayashida T, Jinno H, Hirose S, Sakata M, Takahashi M, Maheswaran S, Mukai M and Kitagawa Y: HOXB9 expression promoting tumor cell proliferation and angiogenesis is associated with clinical outcomes in breast cancer patients. Ann Surg Oncol 19: 1831-1840, 2012.

10. Hoshino Y, Hayashida T, Hirata A, Takahashi H, Chiba N, Ohmura M, Wakui M, Jinno H, Hasegawa H, Maheswaran S, et al: Bevacizumab terminates homeobox B9-induced tumor proliferation by silencing microenvironmental communication. Mol Cancer 13: 102-108, 2014.

11. Wilhelm SM, Adnane L, Newell P, Villanueva A, Llovet JM and Lynch M: Preclinical overview of sorafenib, a multikinase inhibitor that targets both Raf and VEGF and PDGF receptor tyrosine kinase signaling. Mol Cancer Ther 7: 3129-3140, 2008.

12. Liu L, Cao Y, Chen C, Zhang X, McNabola A, Wilkie D, Wilhelm S, Lynch $M$ and Carter C: Sorafenib blocks the $\mathrm{RAF} / \mathrm{MEK} / \mathrm{ERK}$ pathway, inhibits tumor angiogenesis, and induces tumor cell apoptosis in hepatocellular carcinoma model PLC/PRF/5. Cancer Res 66: 11851-11858, 2006.
13. Abate-Shen C: Deregulated homeobox gene expression in cancer: Cause or consequence? Nat Rev Cancer 2: 777-785, 2002.

14. Jain RK: Molecular regulation of vessel maturation. Nat Med 9: 685-693, 2003.

15. Shrestha B, Ansari KI, Bhan A, Kasiri S, Hussain I and Mandal SS: Homeodomain-containing protein HOXB9 regulates expression of growth and angiogenic factors, facilitates tumor growth in vitro and is overexpressed in breast cancer tissue. FEBS J 279: 3715-3726, 2012.

16. Nguyen DX, Chiang AC, Zhang XH, Kim JY, Kris MG, Ladanyi M, Gerald WL and Massagué J: WNT/TCF signaling through LEF1 and HOXB9 mediates lung adenocarcinoma metastasis. Cell 138: 51-62, 2009.

17. Abou-Alfa GK, Schwartz L, Ricci S, Amadori D, Santoro A, Figer A, De Greve J, Douillard JY, Lathia C, Schwartz B, et al: Phase II study of sorafenib in patients with advanced hepatocellular carcinoma. J Clin Oncol 24: 4293-4300, 2006.

18. Newell P, Toffanin S, Villanueva A, Chiang DY, Minguez B, Cabellos L, Savic R, Hoshida Y, Lim KH, Melgar-Lesmes P, et al: Ras pathway activation in hepatocellular carcinoma and anti-tumoral effect of combined sorafenib and rapamycin in vivo. J Hepatol 51: 725-733, 2009. 